\title{
Properties of the matter created in heavy ion collisions - results from the PHOBOS experiment
}

\author{
Krzysztof Woźniak * \\ Institute of Nuclear Physics Polish Academy of Sciences, Kraków, Poland \\ E-mail: krzysztof.wozniak@ifj.edu.pl
}

for the PHOBOS Collaboration:

B.Alver ${ }^{4}$, B.B.Back ${ }^{1}$, M.D.Baker ${ }^{2}$, M.Ballintijn ${ }^{4}$, D.S.Barton ${ }^{2}$, R.R.Betts ${ }^{6}$, A.A.Bickley ${ }^{7}$,

R.Bindel ${ }^{7}$, W.Busza ${ }^{4}$, A.Carroll ${ }^{2}$,Z.Chai ${ }^{2}$, V.Chetluru ${ }^{6}$, M.P.Decowski ${ }^{4}$, E.García ${ }^{6}$, T.Gburek $^{3}$,

N.George ${ }^{2}$, K.Gulbrandsen $^{4}$, C.Halliwell ${ }^{6}$, J.Hamblen ${ }^{8}$, I.Harnarine ${ }^{6}$, M.Hauer ${ }^{2}$,

C.Henderson ${ }^{4}$, D.J.Hofman ${ }^{6}$, R.S.Hollis ${ }^{6}$, R.Hotyński ${ }^{3}$, B.Holzman ${ }^{2}$, A.Iordanova ${ }^{6}$, E.Johnson ${ }^{8}$,

J.L.Kane ${ }^{4}$, N.Khan ${ }^{8}$, P.Kulinich ${ }^{4}$, C.M.Kuo ${ }^{5}$, W.Lit ${ }^{4}$, W.T.Lin ${ }^{5}$, C.Loizides ${ }^{4}$, S.Manly ${ }^{8}$,

A.C.Mignerey ${ }^{7}$, R.Nouicer ${ }^{2}$, A.Olszewski ${ }^{3}$, R.Pak ${ }^{2}$, C.Reed ${ }^{4}$, E.Richardson $^{7}$, C.Roland ${ }^{4}$,

G.Roland $^{4}$, J.Sagerer ${ }^{6}$, H.Seals ${ }^{2}$, I.Sedykh ${ }^{2}$, C.E.Smith ${ }^{6}$, M.A.Stankiewicz ${ }^{2}$, P.Steinberg ${ }^{2}$,

G.S.F.Stephans ${ }^{4}$, A.Sukhanov ${ }^{2}$, A.Szostak ${ }^{2}$, M.B.Tonjes ${ }^{7}$, A.Trzupek ${ }^{3}$, C.Vale ${ }^{4}$,

G.J.van Nieuwenhuizen ${ }^{4}$, S.S.Vaurynovich ${ }^{4}$, R.Verdier ${ }^{4}$, G.I.Veres ${ }^{4}$, P.Walters ${ }^{8}$, E.Wenger ${ }^{4}$,

D.Willhelm ${ }^{7}$, F.L.H.Wolfs ${ }^{8}$, B.Wosiek ${ }^{3}$, K.Woźniak ${ }^{3}$, S.Wyngaardt ${ }^{2}$, B.Wystouch ${ }^{4}$

${ }^{1}$ Argonne National Laboratory, Argonne, IL 60439-4843, USA

${ }^{2}$ Brookhaven National Laboratory, Upton, NY 11973-5000, USA

${ }^{3}$ Institute of Nuclear Physics PAN, Kraków, Poland

${ }^{4}$ Massachusetts Institute of Technology, Cambridge, MA 02139-4307, USA

${ }^{5}$ National Central University, Chung-Li, Taiwan

${ }^{6}$ University of Illinois at Chicago, Chicago, IL 60607-7059, USA

${ }^{7}$ University of Maryland, College Park, MD 20742, USA

${ }^{8}$ University of Rochester, Rochester, NY 14627, USA

In the collisions of ultrarelativistic heavy ions the energy of the nuclei is released in a small volume leading to the creation of a hot and dense nuclear matter. The study of the particles produced from it gives the information on the conditions in the early stage of the collision and the evolution of the system. The PHOBOS experiment provides unique data on the particle production in the almost full phase space and down to very small transverse momenta.

This overview shortly presents the most important features of the nucleus-nucleus collisions observed by the PHOBOS experiment, but concentrates on the most recent studies. The extensive analyses of charged particles production are based on the multiplicity measurements in a very wide pseudorapidity range and reveal strong short and long range correlations. The correlations with a high $p_{T}$ trigger particle extends at least 4 units in pseudorapidity, while two-particle correlations suggest production of particles in large and wide clusters.

European Physical Society Europhysics Conference on High Energy Physics, EPS-HEP 2009,

July $16-222009$

Krakow, Poland

*Speaker. 


\section{Introduction}

The Relativistic Heavy Ion Collider provides since the year 2000 collisions of heavy nuclei at unprecedented energy, $\sqrt{s_{N N}}=200 \mathrm{GeV}$. Conditions occurring in these collisions are sufficient for creation of a new type of dense and hot nuclear matter: strongly interacting Quark-Gluon Plasma (sQGP). Particles, which are produced as a result of evolution of this system and hadronization, exhibit features which were not observed at lower collision energies. The most striking results are the absorption of high momentum partons in the dense matter and strong collective effects observed as the elliptic flow [1].

The PHOBOS experimental setup (for details see [2]) includes the trigger and TOF systems based mostly on scintillators, but the detailed measurements of event properties are possible thanks to spectrometer and multiplicity detector built of silicon sensors. The two-arm spectrometer with 16 layers of sensors is placed in the 2 Tesla magnetic field and enables measurement of particles with momenta as low as $30 \mathrm{MeV} / \mathrm{c}$. The multiplicity detector features one layer of sensors arranged close to the beam pipe into an octagon, near the interaction point, and six rings, at larger distances. Together they register hits from charged particles in almost full solid angle: $|\eta|<5.4$ and full azimuthal angle (except the holes for the spectrometer and for the vertex detector).

The PHOBOS experiment has contributed to the discovery of all important features of the sQGP [3]. It measured the charged particles transverse momentum distributions for all collision systems available at RHIC: $d+A u, C u+C u$ and $A u+A u[4,5,6]$. These measurements clearly show, that the heavier are the colliding nuclei and the more central is the collision, then the less frequently high- $p_{T}$ particles are produced. Such particles originate from the partons created in hard collisions of the constituents of nucleons and can be absorbed in the dense, strongly interacting matter. The path length in it is obviously much longer when the area of the overlap of nuclei is large. This is why in the central $A u+A u$ collisions the scaled yield $R_{A A}=\frac{\sigma_{p \bar{p}}^{\text {inel }}}{N_{c o l l}} \frac{d^{2} N_{A A} / d p_{T} d \eta}{d^{2} \sigma_{p \bar{p}} / d p_{T} d \eta}$ at $p_{T}>4 \mathrm{GeV} / \mathrm{c}$ is almost 5 times smaller than in $d+A u$ collisions [4].

At the other extreme - the smallest transverse momenta - PHOBOS has measured the particle yields for pions $\left(p_{T} \approx 30-50 \mathrm{MeV} / \mathrm{c}\right)$, kaons and protons $\left(p_{T} \approx 100-200 \mathrm{MeV} / \mathrm{c}\right)$ [7]. These yields were found to be consistent with the extrapolations from higher momenta obtained for the models, which assume the expansion of the system created in the collision. There is no evidence of anomalous enhancement of these yields, which was expected for weakly interacting QGP and especially for creation of disordered chiral condensate (DCC).

Finally, the PHOBOS experiment has measured the elliptic flow, for many collision systems, energies and centralities as a function of pseudorapidity or $p_{T}$ of the particles $[3,8,9]$. The large magnitude of this observable indicates that the collective effects are strong. According to hydrodynamic calculations the sQGP has properties of a perfect fluid.

\section{Correlations with a high $p_{T}$ trigger particle}

The source of high-momentum particles are hard scattering of nucleon components (quarks or gluons), in which pairs of back-to-back emitted partons are created. As mentioned above, the yield of the high- $p_{T}$ particles originating from these partons decreases in the central nucleus-nucleus collision, and this induces immediately the question: what happens to the partons, which are the 

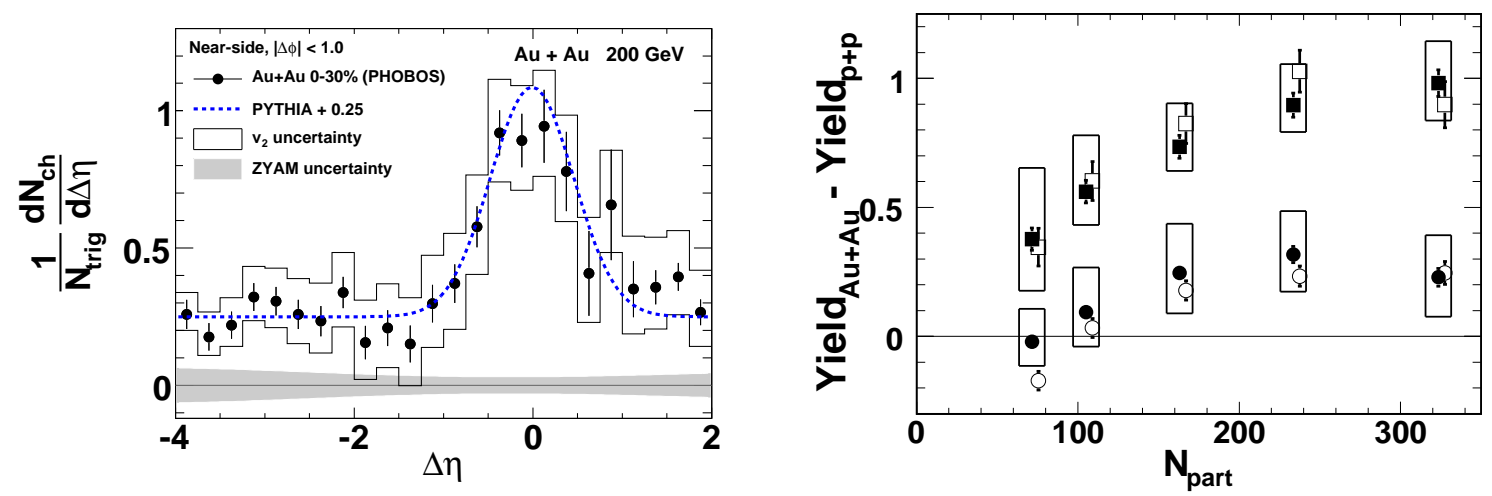

Figure 1: The yield of the particles correlated with a high $p_{t}$ trigger particle in $A u+A u$ collisions at $\sqrt{s_{N N}}=200 \mathrm{GeV}$. (left) An example of the dependence on $\Delta \eta$ in the near-side $(|\Delta \phi|<1)$ for central collisions (0-30\%). (right) The yield from $A u+A u$ collisions (with the yield from $p+p$ collisions subtracted) for all combinations of applied selections: near side ( $\Delta \phi \approx 0$ - circles) or away side ( $\Delta \phi \approx \pi$ - squares) and short range $(|\Delta \eta|<1$ - open symbols) or long range ( $-4<\Delta \eta<-2$ - full symbols), shown as a function of event centrality (represented by the number of nucleons participating in the collision, $N_{\text {part }}$ ).

source of the energetic particles. The answer to it is given by the study of $200 \mathrm{GeV} A u+A u$ collisions, in which charged particles are correlated with a high- $p_{T}$ trigger particle $\left(p_{T}>2.5 \mathrm{GeV} / \mathrm{c}\right)$ from one of the partons. The correlations are measured as a function of $\Delta \eta$ and $\Delta \phi$ - the difference between the pseudorapidities and azimuthal angles of the trigger particle and all remaining charged particles. Measured yields are corrected for the presence of elliptic flow [11, 10]. In a more quantitative analysis we are using projections of the $2 \mathrm{D}$ histogram on one axis. Let's consider first the yield of correlated particles emitted at approximately the same azimuthal angle $(\Delta \phi \approx 0)$ as a function of $\Delta \eta$. As shown in Fig.1(left), for the sample of central $A u+A u$ events it features a peak at $\Delta \eta \approx 0$ and the "ridge" (a non-zero value) extending to a large distance in pseudorapity (at least to $|\Delta \eta| \approx 4$ ). The comparison with the elementary $p+p$ collisions (from PYTHIA generator) shows, that the yield in $A u+A u$ can be described as a constant term (of about 0.25) plus the $p+p$ yield.

Interesting results are obtained also for the yield of correlated particles presented as a function of $\Delta \phi$. We have studied it in two pseudorapidity intervals: short range $(|\Delta \eta|<1)$ and long range $(-4<\Delta \eta<-2)$. The $\Delta \phi$ distribution always features a narrow peak at the near side $(\Delta \phi \approx 0)$ and a broad one on the opposite (away) side. The height and the area under the peaks vary with centrality of the collisions and are different in the short and long range in $\Delta \eta$. Both peaks are the biggest in the most central events and become smaller for more peripheral collisions. The narrow peak at $\Delta \phi \approx 0$ is much higher in the short range than in the long range in $\Delta \eta$, while the broad peak at away side looks similar in both ranges. Much more similar become the results in the short and long range when from the yield in $A u+A u$ collisions the yield from elementary $p+p$ interactions is subtracted. Such surplus yields are shown in Fig.1(right), where four combination of ranges in $\Delta \eta$ and $\Delta \phi$ are included. The enhancement over elementary collisions is practically the same at short and long range, but it is larger at away side than at the near side. For peripheral events all these additional yields decrease, and the near side ridge seems to disappear at about $N_{\text {part }}=80$. 

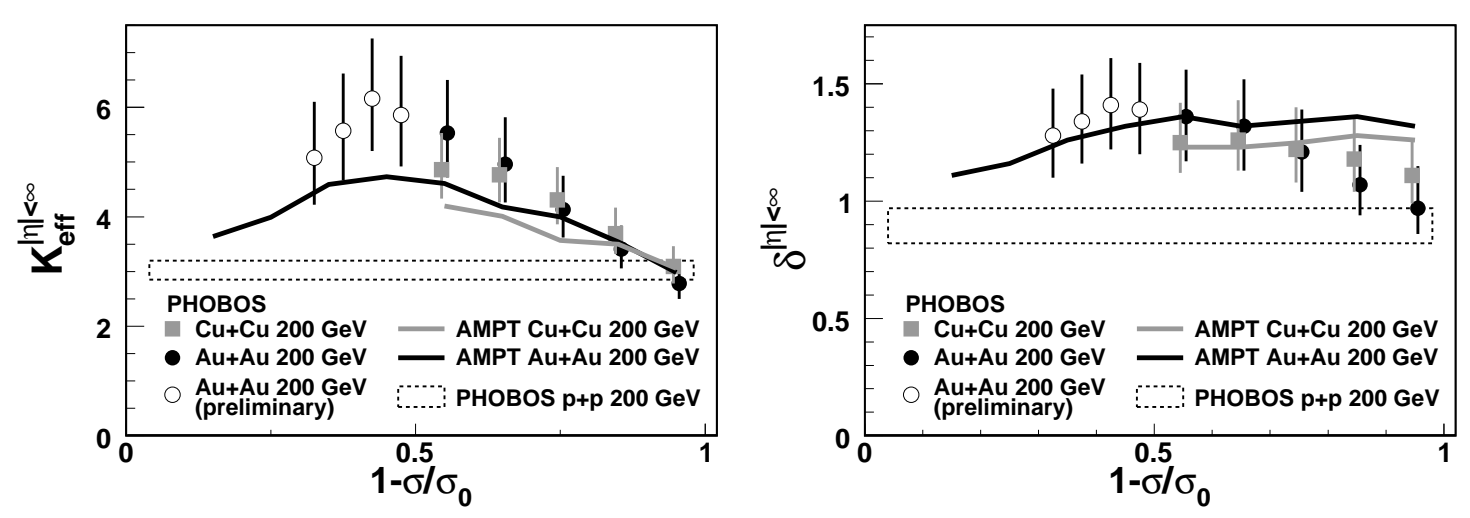

Figure 2: The effective parameters of the clusters corrected for the acceptance effects: the cluster size (left) and the cluster width $\delta$ (right) as a function of the fractional cross section, i.e. obtained for similar geometry of $A u+A u$ and $C u+C u$ collisions. The cluster parameters obtained for elementary $p+p$ collisions [13] and the results of the same reconstruction procedure performed on the events from AMPT generator are shown for comparison.

\section{Two particle correlations}

The nature of the matter created in the collisions of nuclei and especially the properties of the process of final particle production are also reflected in the correlations among all charged particles. In the PHOBOS experiment they are studied using particles from a wide pseudorapidity range $(|\Delta \eta|<3)$ with almost full acceptance in the azimuthal angle. The procedure of this analysis is described in details in Ref. [12]. The two dimensional correlation function, in $\Delta \eta$ and $\Delta \phi$, has a maximum at $(0,0)$ and a broader peak at away side $(0, \pi)$. In the collisions of nuclei the elliptic flow is visible as a sinusoidal enhancement with maxima at $\Delta \phi \approx 0$ and $\Delta \phi \approx \pi$. The integration of the correlation function over $\Delta \phi$ allows to obtain quantitative results not sensitive to elliptic flow or momentum conservation effects.

For elementary $p+p$ collisions the shape of correlation function is very similar to the predictions from the simple cluster model, as shown in Ref. [13], it is thus reasonable to describe the correlations using parameters of clusters. The cluster model simply assumes that production of particles happens in two steps: at first some heavier objects (clusters) are created, which then decay into finally observed particles. The natural candidates for clusters are hadronic resonances, however the model does not assume that only known resonances are allowed. In the original approach [14] the decay is isotropic in the rest frame of the cluster.

The parameters of clusters, which can be directly extracted from the correlation function, are the cluster size $k$ (the number of particles originating from the cluster, for a mixture of clusters with different sizes: $k_{e f f}=\langle k\rangle+\sigma^{2}(k) /<k>$ ) and the cluster width $\delta$ (RMS of the distance between the particles from the cluster). The values of $k_{e f f}$ and $\delta$ have to be corrected for limited acceptance of the detector, as the clusters are effectively smaller and narrower if some particles from them are not detected. The acceptance effects are large even in the case of a very wide pseudorapidity range $(|\eta|<3)$ available in the PHOBOS experiment, the correction factor for the cluster size $k_{\text {eff }}$ may reach 2.5 [12]. As we can see in Fig.2(left) the reconstructed effective cluster size reaches 6 
charged particles, much more than that for known resonances. Also the width $\delta$, up to 1.4 , is larger than the width expected for isotropic decay of any object, even that with negligible $p_{T}$. It clearly indicates, that the correlations can not be of a purely kinematical origin.

For the $A u+A u$ collisions at $\sqrt{s_{N N}}=200 \mathrm{GeV}$ the smallest size $k_{\text {eff }}$ is observed for the most central collisions, it reaches a maximum at semi-peripheral collisions and decreases for the most peripheral, possibly down to the value found for $p+p$ collisions. Interestingly, very similar $k_{e f f}$ and $\delta$ values are obtained also for $C u+C u$ collisions, when they are compared for similar geometry of the collision (i.e. the fractional cross section). This means that the correlations are sensitive not only to the details of hadronization but also to the properties of the early stage of the collision.

\section{Summary}

The PHOBOS experiment delivered data on charged particles production for uniquely large acceptance in pseudorapidity. Recently, they were used in the analysis of two-particle correlations and the correlations with a high $p_{T}$ trigger particle. In both studies strong and long range correlations were found. The particles are produced in large and wide clusters, the ridge found in the correlations with a high $p_{t}$ particle extends at least 4 units in $\Delta \eta$. Different is however the centrality dependence of these effects: the largest clusters are observed for semi-peripheral collisions, while the yield of particles correlated with the trigger particle is the largest for central collisions.

This work was partially supported by U.S. DOE grants DE-AC02-98CH10886, DE-FG02-93ER40802, DE-FG02-94ER40818, DE-FG02-94ER40865, DE-FG02-99ER41099, and DE-AC02-06CH11357, by U.S. NSF grants 9603486, 0072204, and 0245011, by Polish MNiSW grant N N202 282234 (2008-2010), by NSC of Taiwan Contract NSC 89-2112-M-008-024, and by Hungarian OTKA grant (F 049823).

\section{References}

[1] M. Gyulassy, L. McLerran, Nucl. Phys. A750 (2005) 30.

[2] B.B. Back et al., Nucl. Instr. and Meth. A 499 (2003) 603.

[3] B.B. Back et al., Nucl. Phys. A757 (2005) 28.

[4] B.B. Back et al., Phys. Rev. Lett. 91 (2003) 072302.

[5] B. Alver et al., Phys. Rev. Lett. 96 (2006) 212301.

[6] B.B. Back et al., Phys. Lett. B 578 (2004) 297.

[7] T. Gburek et al., J. Phys. G35 (2008) 104131.

[8] B.B. Back et al., Phys. Rev. C 72 (2005) 051901.

[9] B. Alver et al., Phys. Rev. Lett. 98 (2007) 242302.

[10] G.S.F. Stephans et al., arXiv: 0907.4805v1 [nucl-ex].

[11] B. Alver et al., arXiv:0903.2811v2 [nucl-ex] (submitted to Phys. Rev. Lett.).

[12] B. Alver et al., arXiv:0812.1172v1 [nucl-ex] (submitted to Phys. Rev. C).

[13] B. Alver et al., Phys. Rev. C 75 (2007) 054913.

[14] A. Morel and G. Plaut, Nucl. Phys. B78 (1974) 541. 